\title{
Collaborative care models for improving hypertension control through Self-Measured Blood Pressure Monitoring
}

\author{
Margaret Meador \\ Judy Hannan \\ Debosree Roy \\ Kate Whelihan \\ Nana Sasu \\ Heather Hodge \\ Joy H. Lewis
}

\section{Video Abstract}

Keywords: Journal of Community Health, self-measured blood pressure monitoring, SMBP, home blood pressure monitoring, collaborative care models, community health center, hypertension, hypertension control, cardiovascular disease, Centers for Disease Control and Prevention, CDC, YMCA, blood pressure monitoring, community partnership, public health organization, training, outreach, lifestyle, community health workers, clinician

Posted Date: October 12th, 2021

DOI: https://doi.org/10.21203/rs.3.rs-965432/v1

License: (c) (1) This work is licensed under a Creative Commons Attribution 4.0 International License. Read Full License 


\section{Abstract}

Uncontrolled hypertension is a leading risk factor for cardiovascular disease. Despite available treatments, less than half of those with hypertension have optimal blood pressure control, with that percentage continuing to go down. Self-measured blood pressure monitoring (or SMBP) with clinical supports is one effective way of controlling blood pressure. Unfortunately, in the US, as few as $25 \%$ of adults with hypertension measure their blood pressure at home, and even fewer follow an [evidencebased protocol] to take regular home measurements and share them with their care team. Limited use of SMBP is due to a variety of barriers that both patients and their health care provider teams encounter. In search of ways to improve these poor statistics, the Centers for Disease Control and Prevention, the National Association of Community Health Centers, the YMCA of the USA, and the Association of State and Territorial Health Officials conducted a pilot project in nine community health centers across three states. The goal was to increase use of SMBP - the stipulation: it needed to be done using a collaborative model with health centers, community organizations, and health departments. The results are featured in a recent Journal of Community Health article. Over the course of 17 months, nine health centers partnered with seven local Ys and local health departments, as well as other community partners, to generate nine collaborative care models for improving SMBP uptake among adults with hypertension. Although the models were uniquely shaped by available community assets, strategic priorities, and organizational culture, they had four components in common: first, a focus on building community partnerships to implement SMBP as a tool for hypertension care; second, determining SMBP tasks that can be carried out by people other than the clinical provider or patient; third, leveraging the expanded care team and/or community and public health resources to carry out these tasks; and fourth, establishing regular knowledge-sharing sessions to make refinements and identify best practices. As a result of these efforts, 1,421 patients with uncontrolled hypertension were identified and recommended for SMBP, with 795 completing at least one cycle of SMBP. One cycle was defined as blood pressure measurements morning and evening for at least three consecutive days. Integrating self-measured blood pressure monitoring into routine hypertension care is critical. Collaborative partnerships between care delivery organizations, public health agencies, and community organizations, like the $Y$, mitigate capacity barriers and increase support to patients using SMBP. Moreover, SMBP may serve as an entry activity to connect clinic activities and community supports more broadly. 